\title{
¿DERECHO A PRIORI VS. DERECHO NATURAL? LA CONTRIBUCIÓN DE ADOLF REINACH
}

\author{
A PRIORI LAW (UNDERLYING JURIDICAL ELEMENTS) VS. NATURAL \\ LAW? THE ADOLF REINACH'S CONTRIBUTION
}

\section{MARIANO CRESPO*}

No es difícil constatar cómo el derecho positivo se halla en cambio y desarrollo permanentes. Leyes que no se adaptan a las nuevas circunstancias sociales son abolidas mientras que otras son promulgadas a fin, precisamente, de dar respuesta a esas nuevas circunstancias. La constatación de este cambio lleva a plantearse la cuestión de si esto es todo. Dicho con otras palabras: ¿significa la diversidad del derecho positivo que las formaciones específicamente jurídicas (piénsese, por ejemplo, en la propiedad, la pretensión, la obligación, la representación, etc.,) no tienen un ser independiente del acto del legislador, en particular, y de los hombres que las comprenden, en general? Dicho con otras palabras, ¿son estas formaciones, en última instancia, creaciones del derecho positivo como lo son las diversas leyes emanadas del correspondiente poder legislativo?

Una respuesta negativa a esta pregunta es la representada por las diversas teorías iusnaturalistas en cuanto que ponen de relieve la idea de un derecho de validez incondicionada el cual debe dar, bajo todas las circunstancias, un punto de dirección para el legislador. Las normas que contravengan este derecho serían injustas y carecerían de carácter vinculante, aun cuando hayan sido promulgadas por la autoridad competente cumpliendo los diversos requisitos formales.

Ahora bien, llegados a este punto, se nos plantea una nueva cuestión: ¡es el iusnatu-

\footnotetext{
* Licenciado en Filosofía, Doctor en Filosofía por la Universidad Complutense de Madrid, profesor adjunto Ordinario de la Facultad de Filosofía de la Pontificia Universidad Católica de Chile, mail de contacto: mcrespos@uc.cl
}

ralismo la única respuesta a la pregunta que nos había suscitado la constatación del cambio y desarrollo permanentes del derecho positivo? Expresado de otra forma, ¿no habría otro modo de poner de manifiesto la objetividad de ciertos elementos subyacentes al derecho positivo? En este orden de cosas, parecería que la idea de la doctrina apriorística del Derecho formulada por Adolf Reinach en su obra Los fundamentos apriorísticos del Derecho civil (Trad. De J.L. Álvarez, Librería Bosch, Barcelona 1934) constituiría una respuesta positiva a esta pregunta. La tesis de Reinach en este libro es que frente a la variabilidad del Derecho positivo, las formaciones jurídicas como, por ejemplo, las promesas que están en la base de los contratos, poseen un ser independiente de este derecho "igual que los números poseen un ser independiente de la ciencia matemática" (op. cit., p. 27). Así, que una pretensión surgida de una promesa se extingue por el cumplimiento es tan evidente como un axioma lógico o matemático.

De este modo, se abre aquí un nuevo campo a filosofía. En cuanto ontología o teoría a priori del objeto esta se encuentra con una especie completamente nueva de objetos, "objetos que no pertenecen a la Naturaleza en el sentido propio, que no son físicos ni psíquicos, y que a su vez difieren también de todos los objetos ideales: por su temporalidad" (ibid.). Dos son las notas fundamentales que según Reinach caracterizan a estos objetos, a saber, su "ser-así"-y-no-poder-ser-de-otromodo o, lo que es lo mismo, su necesidad, y su carácter a priori. Expliquemos brevemente en qué consisten estas notas.

En primer lugar, la necesidad a la que Reinach se refiere no es una necesidad psicoló- 
gica. No se trata, pues, de una consecuencia del "tener que pensar así" y del "no poder pensar de otra manera" enraizada, en última instancia, en nuestra peculiar constitución psicológica, sino de una necesidad de lo pensado. Tampoco es una necesidad de la naturaleza como la que observamos en las leyes de las ciencias naturales, obtenidas estas últimas a través de la constatación real y de la inducción, la cual a lo sumo arroja alta probabilidad, pero no certeza absoluta.

Por otra parte, la necesidad esencial a la que Reinach se refiere no se restringe a la relación entre el universal y el particular, sino que se trata de una necesidad interna, estructural, que está presente tanto en el estado de cosas general como en el individual. Esta necesidad es mucho más primaria de modo que podemos decir que la necesidad formal del dominio de la naturaleza universal sobre el caso individual se funda en ella.

En segundo lugar, los objetos o entidades de los que venimos hablando son caracterizados por Reinach como a priori o aprióricos. Así se habla, por ejemplo, de una teoría a priori o apriorística de los objetos. En la jerga filosófica a priori y a posteriori son términos que suelen utilizarse para referirnos al modo en que algo es conocido. Así, en términos generales, una proposición es a priori cuando es conocida con independencia de la experiencia mientras que una proposición a posteriori es aquella cuyo conocimiento está basado en la misma. Por consiguiente, un esclarecimiento del sentido de estos dos términos requiere la aclaración previa de qué se entiende por "experiencia”. Para ello me serviré de las investigaciones de uno de discípulos de Reinach, Dietrich von Hildebrand.

Con el término "experiencia" se puede mentar, por un lado, el contacto real con el ente aquí y ahora existente y, por otro, la experiencia del "ser así" de aquello que nos es dado. La tradición habría utilizado el término "experiencia" para referirse indistintamente a estos dos elementos. De lo que se trata es de darse cuenta de que la experiencia, en el sentido de constatación real, me permite el acceso solo a la universalidad empírica, pero no a la universalidad absoluta.
Si profundizamos en estos dos sentidos de experiencia, nos encontramos con una semejanza y con una diferencia entre ambos. Por lo que se refiere a la similitud, nos damos cuenta de que en ambos casos la percepción constituye el punto de partida. Sin embargo, ello no nos debe hacer pasar por alto la diferencia que existe entre ambos modos de contacto cognoscitivo. Es cierto que cuando un objeto se nos da a sí mismo, tenemos un conocimiento de su "ser-asî" y también de su existencia real. No obstante, estos dos tipos de experiencia son radicalmente distintos. Mientras que una observación puede ser invalidada porque se trate de una alucinación, la experiencia del "ser-así" no se ve afectada por ello.

Por consiguiente, la experiencia del "ser-así" abre un amplio campo de posibilidades. En este sentido, yo puedo tener experiencia de un acto de bondad sin haber entrado en contacto real con un acto bueno existente. De esta manera, a través de la lectura de una novela o de la consideración de un acto bueno puedo "experimentar" el "ser-así" de la bondad moral. Incluso podría tener experiencia de la bondad a través del conocimiento de su opuesto, a saber, la maldad.

Si tenemos en cuenta los dos sentidos del término "experiencia" que acabamos de analizar (constatación real de las cosas y experiencia del "ser-así"), la proposición "lo a prio$r i$ es independiente de la experiencia" puede tener dos sentidos:

a) Puede indicar independencia de la experiencia en el sentido de independencia de la constatación real de la existencia de algo. La cuestión que aquí se nos plantearía es si podemos conocer un estado de cosas absolutamente cierto y esencialmente necesario con independencia de la experiencia en este sentido. Cuando aquí hablamos de conocimiento a priori no nos estamos refiriendo a la posibilidad, considerada en sí misma, de un conocimiento absolutamente cierto de estados de cosas altamente inteligibles y esencialmente necesarios. Lo que aquí es importante notar es que la independencia de la constatación real no garantiza automáticamente la inteligibilidad de los estados de cosas. 
b) En segundo lugar, "lo a priori es independiente de la experiencia" puede significar independencia de la experiencia del "ser-así". Formulado de otra manera, lo que aquí se debate es la posibilidad del conocimiento de ciertos contenidos con independencia de toda experiencia. Hildebrand aborda este problema de un modo, en mi opinión, muy breve en ¿Qué es filosofía? Allí se atiende a dos casos diferentes. Por un lado, se estudia lo que sucede, por ejemplo, con los colores. En este caso parece evidente que estamos tratando con contenidos que tienen que haberse presentado a la mente humana al menos una vez en su "serasí". En este sentido, un ciego de nacimiento no podría asentir a la proposición que afirma que el naranja es un color que en la escala cromática está entre el rojo y el amarillo. Ahora bien, ¿sucede lo mismo con contenidos como "unidad" o con valores morales básicos como "bueno" o "malo"?

A mi modo de ver, resulta evidente que podemos conocer con certeza absoluta ciertos estados de cosas esencialmente necesarios con independencia de la constatación real. Sin embargo, resulta más difícil pensar en la captación de este tipo de estados de cosas si el objeto en el que estos tienen su raíz no se ha "desplegado" de algún modo ante nuestra mente. ¿Cómo podría yo, por ejemplo, conocer que el correlato objetivo de la proposición "los valores morales se encarnan en personas" es esencialmente necesario si no he tenido un cierto acceso, más o menos tematizado, al "serasî" que es un valor moral? Precisamente si calificamos a este estado de cosas como esencialmente necesario es justamente porque lo percibimos como fundado en la esencia de un determinado tipo de "ser-asî". Por otra parte, la experiencia del "ser-así" constituye un recurso cognoscitivo absolutamente decisivo en la filosofía.

Dicho lo dicho, se comprende que Reinach no aplique el término a priori en sentido primario a una determinada clase de conocimientos o a la forma de ciertas proposiciones sino a "lo dado", a la esfera de los hechos, y una proposición es solo verdadera a priori (o falsa) en cuanto que se cumple en tales hechos Por consiguiente y tal como Reinach lo men- ciona en su estudio de la interpretación kantiana de Hume, lo que merece el nombre de $a$ priori en primer y auténtico sentido son determinados estados de cosas, "aquellas entidades peculiares que hemos de diferenciar cuidadosamente de toda objetividad que en ellas entre como elemento y de todo juzgar y conocer que se refiera intencionalmente a ellos".

Ello determina que todo aquello que vale para la esencia de estos vale también a priori para todos los objetos de esa esencia. Por consiguiente, la esfera de lo a priori no se reduce a lo formal, sino que también hay un $a$ priori material que encontramos, por ejemplo, en la teoría de los números, la teoría de las cantidades, la teoría de grupos, la geometría, la mecánica, la física, la química, la biología, la psicología, el derecho, etc. De este modo, en todas estas ciencias nos encontramos con todo un sistema de proposiciones materialmente apriorísticas basadas sobre la intuición de la esencia de los objetos de cada una de ellas.

Resumiendo, cuando Reinach habla de juicios y conocimientos aprióricos la palabra a priori se usa en un sentido derivado. Son a priori los juicios que se refieren a estados de cosas a priori. De hecho, la tarea fundamental de la filosofía consiste en el estudio de estas objetividades aprióricas. Por consiguiente, aquello que realmente distingue el conocimiento a priori del conocimiento empírico es la profunda diferencia en los objetos mismos, entre objetos que poseen una unidad necesaria inteligible y otros que poseen una unidad contingente. Por su parte, los estados de cosas empíricos (sean correlatos de juicios universales inductivos como, por ejemplo, "el calor dilata los cuerpos", sean correlatos de juicios particulares de observación como "hoy hace un calor asfixiante") se nos dan como dependientes de que hay de hecho seres reales concretos. En cambio, los estados de cosas esencialmente necesarios se dan como subsistentes con independencia de que existan objetos para los que valgan. Este hecho, a saber, el que estos estados de cosas valgan con independencia de que exista lo real concreto lo expresamos diciendo que les corresponde aprioridad con respecto al mundo de lo fáctico. Por consiguiente, la 
aprioridad no es una propiedad de los juicios, sino de ciertos estados de cosas. Derivativamente, denominamos a priori al conocimiento de estos estados de cosas.

El esclarecimiento de las entidades $a$ priori en cuanto categoría a la que pertenecen los objetos de una doctrina apriorística del derecho, tal y como es concebida por Reinach, nos permite ofrecer una respuesta positiva a la cuestión que nos planteábamos al inicio de esta intervención. Por lo que se ve, el iusnaturalismo no sería, pues, la única respuesta a la pregunta que nos había suscitado la constatación del cambio y desarrollo permanentes del derecho positivo. La teoría a priori del derecho desarrollada por Reinach constituiría otro modo de poner de manifiesto la objetividad de ciertos elementos subyacentes al derecho positivo. Aquí ambas teorías convergerían como el propio Reinach reconoce claramente:

"Con harta razón suponían los iusnaturalistas que para la fuerza vinculante de los contratos no se necesita una posición determinante de los factores estatales o de otra especie. Con sobrada razón hablan de conexiones jurídicas que existen y pueden investigarse independientemente de la existencia y de la investigación del Estado y de sus determinaciones positivas".

A estas alturas de nuestra exposición podría realizarse una más que justificada pregunta. ¿Existen diferencias sustanciales entre las teorías iusnaturalistas y la doctrina a priori del derecho defendida por Adolf Reinach? La respuesta del propio Reinach es claramente afirmativa. En una, a mi juicio, apresurada y confusa, crítica al iusnaturalismo nuestro autor hace una serie de reproches a las teorías del derecho natural que parecen girar en torno a una supuesta excesiva orientación de este al Derecho positivo. Así el Derecho natural -siempre según Reinach- "rellenaría" los huecos del derecho positivo con el "derecho ideal" o "derecho racional" pero, sobre todo, parecería perder de vista algo a lo que el maestro de Reinach, Edmund Husserl, se había referido en el parágrafo 14 de sus Investigaciones lógicas, a saber, que todas las disciplinas normativas tienen por fundamento disciplinas teoréticas. Así las proposiciones de las primeras, esto es, las proposiciones que expresan lo que debe ser, son susceptibles de ser "traducidas" en proposiciones teoréticas desprovistas ya del giro normativo en cuanto que expresan pura y simplemente lo que es. Así la proposición "un guerrero debe ser valiente" significa en última instancia que solo un guerrero valiente es un buen guerrero y que un guerrero que no sea valiente será un mal guerrero. Como señala Husserl, porque este juicio de valor (el cual ya no es normativo) es válido, tiene razón todo aquel que exija de un guerrero que sea valiente. En general, podemos considerar como iguales, o al menos como equivalentes estas fórmulas: "un A debe ser B" y "un A que no es $B$ es un mal A" o "solo un A que es $B$ es un buen A".

Parecería, por tanto, que, a juicio de Reinach, las teorías del Derecho natural tendrían un interés predominantemente normativo en cuanto que este Derecho constituiría bajo todas las circunstancias, un punto de dirección para el legislador. Este punto de vista viene avalado por una cita que hace Reinach de la Holtzendorffs Enzyklopädie der Rechtswissenschaft en la que el Derecho natural es descrito como "un derecho independiente de las formulaciones humanas y que en ellas solo aparece imperfecto; que tiene su base en una más alta ordenación moral del mundo y de la vida y que está destinada a servir como línea directriz para la crítica y el desarrollo existente". Esta orientación predominantemente normativa se reflejaría asimismo en el hecho de que -siempre según Reinach- no ha sido opinión de todos los iusnaturalistas que el Derecho natural tenga que dar también instrucciones al juez y no solo al legislador. Por su parte, la doctrina apriorística del derecho no hablaría de un "derecho más elevado" sino, cito a Reinach, de "simples leyes del ser" o de leyes fundadas en la esencia de los actos y en la esencia de las formaciones jurídicas.

Los límites de esta ponencia y de este ponente me impiden profundizar en esta cuestión. No obstante, déjenme esbozar tan solo un par de críticas a lo que acabo de exponer.

1. En primer lugar, y aun corriendo el riesgo de caer en el mismo defecto que creo ver en la crítica de Reinach, creo que su crítica 
a las teorías del Derecho natural es un tanto superficial. Ciertamente, hablar de "derecho" natural significa moverse en el ámbito de una disciplina normativa. Sin embargo, la otra cara del Derecho natural es una clara "teoría" acerca, por ejemplo, de la naturaleza humana. Por eso, y en cuanto que la doctrina apriorística del Derecho, busca una serie de contenidos objetivos que han de subyacer al derecho positivo, pienso que esta está más cerca de las teorías del Derecho natural de lo que el mismo Reinach piensa.

2. En segundo lugar, creo que dentro de esos objetos a priori de los que se ocupa la filosofía en cuanto teoría a priori de los objetos en general habría que hacer algunas distinciones más en lo que se refiere a la necesidad de los mismos. Así ciertos derechos a priori -basados por tanto en determinadas formaciones jurídicas a priori- no parecen tener el mismo grado de necesidad que algunos derechos naturales. Así la pretensión que tiene el destinatario de una promesa de que esta se le cumpla por parte del que la ha efectuado puede ser suprimida por el legislador o por un juez cuando esta es realizada, por ejemplo, por un menor de edad o cuando atenta contra ciertos valores morales objetivos. Sin embargo, un derecho natural como el derecho a la vida no puede ser suprimido o anulado por legislador o juez alguno. 